\title{
Evaluation of Participating Media Models for Fire Simulation
}

\author{
P.S. Cumber and ${ }^{\dagger} M$. Fairweather \\ BG Technology, Gas Research \& Technology Centre, Loughborough LE11 3GR, UK \\ ${ }^{\dagger}$ School of Process, Environmental and Materials Engineering, University of Leeds, Leeds \\ LS2 9JT, UK
}

\begin{abstract}
This work considers the application of participating media models, used in conjunction with the discrete transfer method, for predicting thermal radiation fluxes from fires. The models considered differ in their generality, sophistication, accuracy and computational cost, and are assessed in terms of their ability to predict radiative transfer in idealised situations, as well as from fires. It is concluded that the implementation of simple participating media models, based on the grey gas assumption, must be treated with caution due to convergence problems. Use of models based on the banded grey gas, TTNH, wide and narrow band approaches yield satisfactory results for fire applications. Computations performed using Cartesian meshes demonstrate that the TTNH and banded grey gas models give speed-up factors of one and two orders of magnitude relative to more complex approaches, although this level of performance is reduced when more complex meshes are employed.
\end{abstract}

KEYWORDS: radiative heat transfer, participating media models, discrete transfer method

\section{NOMENCLATURE}

$K_{a}=$ absorption coefficient

$l=$ path length

$n=$ number of control volumes

$N=$ number of rays

$q=$ heat flux

$r=$ radial distance

$S=$ speed-up factor
$T \quad=$ temperature

$x \quad=$ axial or downwind distance

$Y \quad=$ mass fraction

Subscripts:

$\mathrm{CO}_{2}=$ carbon dioxide

$\mathrm{H}_{2} \mathrm{O}=$ water vapour

$j \quad=$ gaseous species 


\section{INTRODUCTION}

The safe design and operation of high-pressure plant and pipe work requires that provision be made for the relief of pressure under certain operational and emergency conditions. The consequences of a release must also be evaluated so that appropriate safety measures can be adopted during the relief process. In addition, assessments of the consequences associated with accidental releases of flammable material are required as the basis of safety reports and risk assessments on existing and proposed installations.

For flammable gases and vapours it is necessary to be able to predict the thermal radiation fluxes that any fire might impose on its surroundings - either by direct flame impingement of the fire on an item of plant or at distance from the fire by radiation transmitted through the atmosphere. This information is in turn used to provide estimates of such things as vessel survival times, building burning distances and escape times for personnel.

Predictions of the structure of fires using either integral [1] or numerical [2] techniques require that some representation be made of the absorption and emission characteristics of the products of combustion to allow solution of the radiative transfer equation. In particular, participating media models are required for the gaseous species and unburned carbon particulates that occur within the fire. The present work focuses on the non-luminous radiation attributable to gaseous species since the majority of mathematical models used to calculate radiative heat transfer from fires employ similar techniques [3] for describing the continuum radiation from soot particles, whilst the banded radiation from gaseous products is in general handled using a wide variety of methods. In addition, gaseous emissions contribute significantly to the levels of thermal radiation from and within fires, particularly in the case of fires from high velocity releases. As an example, recent experimental and theoretical work [2] which considered a number of jet fires (up to $2.7 \mathrm{GW}$ in size) stabilised on subsonic releases of natural gas demonstrated that the contribution from soot particles to total radiative fluxes measured about these fires was at most $40 \%$.

The work described considers the application of a number of participating media models, used in conjunction with the discrete transfer method [4] for solving the equation of radiative transfer, to predict radiative fluxes from fires. The latter technique has been adopted due to its frequent use in fire modelling codes, as well as its computational economy, ease of implementation and conceptual simplicity [4]. The participating media models considered differ in their generality, sophistication, accuracy and computational cost, and are each assessed in terms of their ability to predict radiative transfer from one-dimensional, idealised representations of the internal structure of non-premixed flames, as well as from laboratory and field scale jet fires. Of particular interest in the present work is the accuracy and computational cost of the various modelling approaches. In particular, the application of fire models in safety reports and risk assessments currently demands that predictions be made, for example, of the two- and three-dimensional received radiation flux distributions about a fire, which in turn allow time integrated fluxes for personnel following an escape route to a place of safety to be established. This type of calculation is particularly demanding, and the present work considers the appropriate choice of participating media model to achieve the optimum balance between model accuracy and computational cost. 


\section{RADIATIVE HEAT TRANSFER MODELS}

\section{Participating Media Models}

The participating media models employed in the present work were:

- the method due to Modak [5],

- the mixed grey gas model developed by Truelove [6],

- a banded implementation of Truelove's [6] mixed grey gas model,

- the total transmittance, non-homogeneous (TTNH) model of Grosshandler [7],

- a spectral version of the exponential wide band model developed by Edwards and Balakrishnan [8], and

- the statistical narrow band model of Grosshandler [7].

Modak's [5] method is a simple and accurate technique for computing the emissivities and absorptivities of isothermal, homogeneous mixtures of $\mathrm{CO}_{2}$ and $\mathrm{H}_{2} \mathrm{O}$ based on curve fits to emissivities determined using a spectral method. The mixed grey gas model of Truelove [6] is based on representing the banded spectra of $\mathrm{CO}_{2}$ and $\mathrm{H}_{2} \mathrm{O}$ as a mixture of clear and grey gases, with the clear component corresponding to the non-emitting regions of the spectrum between strong gas bands. The calculations reported below employed the four-term, one clear and three grey gas, expansions fitted by Truelove [6] to gas mixture total emittance (for the combined emissions of $\mathrm{CO}_{2}$ and $\mathrm{H}_{2} \mathrm{O}$ ) evaluated from spectral data. Extensions of this method, described by Truelove [6], to incorporate $\mathrm{CO}$ and $\mathrm{CH}_{4}$ emissions were also implemented in the present work, and calculations were performed using fits based on the most appropriate partial pressure ratio for the combined emission. It is also possible to implement the mixed grey gas method in a banded form where, for a given model spectrum, the grey gas weightings are determined as black body fractional functions for specific subline spectral regions. The method employed again used a one clear and three grey gas representation, with the weighting functions taken from Truelove [6]. Further details of this technique may be found elsewhere [9].

The TTNH model $[7,10]$ for $\mathrm{CO}_{2}$ and $\mathrm{H}_{2} \mathrm{O}$ mixtures is based on total transmittance data for homogeneous gases, with effective pressure-path lengths and temperatures for nonhomogeneous systems taken as gas concentration weighted averages along a line-of-sight. The temperature correction factor to the transmittance suggested by these authors $[7,10]$ was not considered in detail in the present work due to its limited range of applicability, although its use is considered further below. The spectral version of the exponential wide band model developed by Edwards and Balakrishnan [8], described in detail by Edwards [11], is based on the fact that the absorption and emission of radiation by a molecular gas is concentrated in between one and six wide vibrational bands. Within these bands, the spectral lines associated with rotational modes of energy storage are re-ordered in wave number space with exponentially decreasing line intensities moving away from the band head. The band shape is then approximated by one of three simple exponential functions, with the radiative properties of each absorption band obtained from specified model parameters. Band overlap is also taken into account for multiple bands and mixtures of gases. The version of this model implemented accounted for mixtures of $\mathrm{CH}_{4}, \mathrm{CO}, \mathrm{CO}_{2}$ and $\mathrm{H}_{2} \mathrm{O}$. Lastly, the narrow band model of Grosshandler [7] is based on the Goody statistical model [12] for triatomic molecules with equal line strengths within each narrow band region, and with non- 
homogeneous effects accounted for through the Curtis-Godson [12] approximation which employs suitable averages along a line-of-sight.

All the models employed were implemented in their standard form and precisely as described by the various authors noted above. Although numerous techniques are available to improve the range of applicability, accuracy and computer run time of many of these methods, these were not pursued in order to ensure a base-line comparison of the various approaches, and to simulate the way in which most researchers use these models.

\section{Discrete Transfer Method}

Numerical solutions to the equation of radiative heat transfer were derived using the discrete transfer method [4]. In its original formulation, this method expresses the transfer equation in a form applicable to homogeneous and grey media. The latter equation is then used to derive a recurrence relation which is applied repeatedly along a ray passing through the homogeneous control volumes defined by the numerical solution procedure for the fluid dynamic equations. By applying this relation to each control volume in turn, inhomogeneities in the overall flow field are accounted for and the total intensity of radiation incident at the end point of the ray determined. The net radiative flux at a given point is then obtained by repeating this calculation for a number of representative rays which are all incident at that point. Extension of this method to non-grey systems using the mixed grey gas approach and applying the recurrence relation over a number of wavelength bands has been described by Lockwood and Shah [4]. Further extensions to allow the incorporation of the spectral version of the wide band model have been described by Cumber et al. [13].

In obtaining the results described below the discrete transfer method was used either in conjunction with specified gas temperature and species profiles, or with local values of the latter parameters and path lengths determined from solutions to the fluid flow equations obtained using a numerical model described in detail elsewhere [2, 13]. The latter model was based on solutions of the density-weighted, averaged transport equations, with closure achieved using a standard $k-\varepsilon$ turbulence model. The gas-phase, non-premixed combustion process was modelled by assuming fast chemical reaction via the conserved scalar/prescribed probability density function (p.d.f.) approach using the laminar flamelet concept. A two-parameter, $\beta$-p.d.f. was used, with the form of this p.d.f. being specified in terms of the mean and variance of mixture fraction obtained from solution of modelled transport equations. Solution of the transport equations was achieved using a finite-volume technique based on the pressure correction algorithm, with path lengths and local mean gas temperatures and species concentrations required for the radiation calculations taken from the homogeneous control volumes used as the basis of this method.

In interfacing the discrete transfer method with numerical solutions to the fluid flow equations, predictions along each ray which employed the participating media model of Modak [5], the banded and non-banded versions of the mixed grey gas model, and the exponential wide band model were obtained using the recurrence relations given by Lockwood and Shah [4] and by Cumber et al. [13]. For the TTNH and narrow band approaches, non-homogeneous profiles, specified at a number discrete points corresponding to local control volume values, were input directly to the expressions used to give weighted averages along a line-of-sight. In the results given below, predictions of radiative transfer 


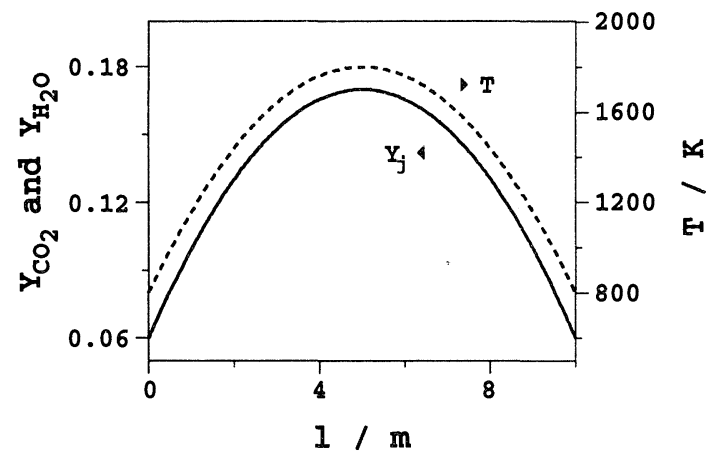

FIGURE 1 Temperature and composition distributions for the one-dimensional test case.

were obtained using mean flow properties, i.e. the influence of turbulence-radiation interactions was ignored. These effects are, however, anticipated to be small [2] in the types of fire considered in the following section.

\section{RESULTS AND DISCUSSION}

In evaluating the various participating media models used in conjunction with the discrete transfer method, it is first useful to consider an idealised, one-dimensional situation representative of the non-homogeneous paths encountered in fires. Figure 1 shows temperature and mass fraction profiles for a path similar to that considered by Grosshandler [7]. These profiles may be considered representative of the radial temperature and concentration distributions that are found downstream of the source in jet fires.

Figure 2 gives results derived from the various models for the profiles of Figure 1. In obtaining these predictions, the profiles were represented by a number of equal path length, homogeneous cells, and the discrete transfer method applied to the three-dimensional control volumes thereby constructed. A total of eight rays was used for each calculation, with this number of rays having been found to give converged predictions of received heat flux. This approach was employed in order to determine received fluxes rather than the less meaningful radiative intensities that would be derived from strictly one-dimensional computations. Results were obtained by doubling the number of control volumes (the abscissa of Figure 2) used to represent the non-homogeneous profiles from 1 to 256 , thereby improving the representation of inhomogeneities along the line-of-sight.

Results derived using the banded version of the mixed grey gas model, the TTNH method, and the wide and narrow band approaches all converge to constant values with increasing numbers of control volumes. This behaviour is essential if reliable predictions of radiative heat transfer are to be obtained in computational fluid dynamic calculations of fires since, once the non-homogeneous profiles are represented to a sufficient level of accuracy by the homogeneous control volumes of such a computation method, integration of the radiative transfer equation along that path must return a fixed intensity value. 


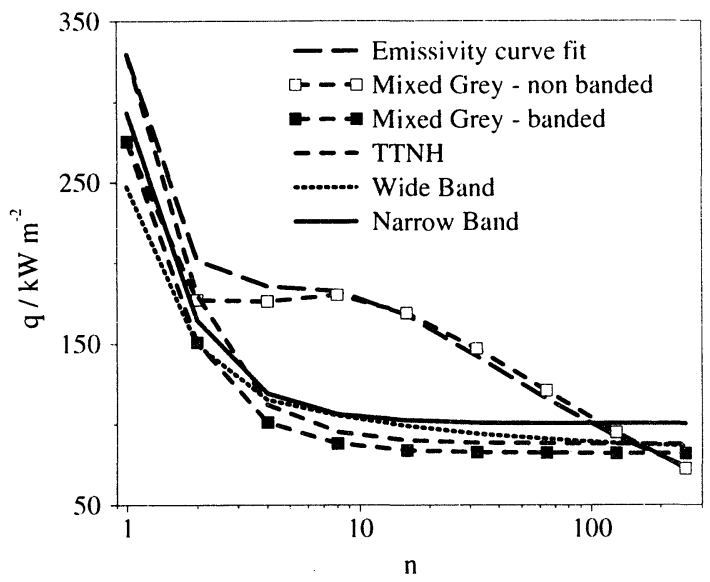

FIGURE 2 Variation of received flux with increasing numbers of control volumes for the one-dimensional test case.

Predictions derived using the emissivity curve fit method [5] and the non-banded, mixed grey gas approach are similar. They do not, however, converge to constant values, with radiative fluxes decreasing dramatically as the representation of the non-homogeneous path is refined. Similar computations performed for homogeneous mixtures also demonstrated that predicted fluxes increase continuously with increasing numbers of control volumes. This occurs since in using these techniques with the recurrence relation of the discrete transfer method a mean absorption coefficient, obtained from the local emissivity, must be specified for each control volume. As path length is reduced, however, the mean absorption coefficient obtained increases, and does not tend to zero. This in turn implies that as the computational mesh is refined the sensitivity of the predicted intensity field (or fluxes) to local path length increases. This convergence problem, caused by the assumption of grey gas behaviour, is well known in the radiation modelling community, but applications of this type of methodology, in conjunction with numerical solutions to the fluid flow equations, still appear in the literature. These results do not, however, imply that the emissivity curve fit method [5] and the non-banded, mixed grey gas model cannot be applied in fire computations to yield reliable results. The results and conclusions reached here are a function of the way in which these models have been implemented in conjunction with the discrete transfer method. Nevertheless, computations based on the current implementation abound in the literature.

Table 1 gives speed-up factors, relative to run times of the narrow band approach, for the participating media models that gave converged results for the one-dimensional test case, with the factors quoted being for received fluxes which varied by less than $2 \%$ when the number of control volumes was doubled. As might be anticipated, the run times of the wide and narrow band approaches far exceed those of the alternative methods due to the intensive nature of detailed spectral calculations. Surprisingly, however, the run time of the wide band approach was significantly greater than that of the more intensive narrow band model, 
TABLE 1. Speed-up factors for various participating media models.

\begin{tabular}{|l|c|c|c|c|c|}
\hline \multirow{2}{*}{$\begin{array}{c}\text { Media } \\
\text { Model }\end{array}$} & \multicolumn{2}{|c|}{$\begin{array}{c}\text { One-Dimensional Test } \\
\text { Case }\end{array}$} & $\begin{array}{c}\text { Laboratory } \\
\text { Scale Fire }\end{array}$ & \multicolumn{2}{c|}{ Field Scale Fire } \\
\cline { 2 - 6 } & $n$ & $S$ & $S$ & $N$ & $S$ \\
\hline $\begin{array}{l}\text { Mixed Grey } \\
\text {-banded }\end{array}$ & 32 & 309.2 & 25.9 & 416 & 273.1 \\
\hline TTNH & 16 & 142.7 & 20.2 & 465 & 75.4 \\
\hline Wide Band & 128 & 0.6 & 2.8 & 423 & 4.1 \\
\hline $\begin{array}{l}\text { Narrow } \\
\text { Band }\end{array}$ & 16 & 1 & 1 & 408 & 1 \\
\hline
\end{tabular}

primarily due to the large number of control volumes required to give converged results. The mixed grey gas, TTNH and narrow band approaches all require relatively small numbers of control volumes to represent the non-homogeneous profiles to a sufficient degree of accuracy, with the former methods giving speed-up factors, respectively, of more than 300 and almost 150 relative to the narrow band model. However, only sixteen control volumes were required by the wide band model to give fluxes which varied by less than $5 \%$ when $n$ was doubled.

Although the comparisons considered so far are instructive, they are not representative of how a radiation model would generally be used in practice. In computations of fires, therefore, a grid-independent, numerical solution for the internal structure of the fire would (at some stage) be made available to the radiation calculation, either as part of a coupled fluid flow-radiative transfer procedure, or with received fluxes determined by postprocessing. In either case, once the ray tracing element of the discrete transfer method has been performed, the number of homogeneous cells used to represent a non-homogeneous path through the fire would be fixed, with the path length through each homogeneous control volume also determined by the ray tracing algorithm. This information would ordinarily be used without further modification by the radiation model.

Numerical calculations using the fire structure model described earlier were made for two flames of widely differing scales. First, for the laboratory scale methane flame considered by Baillie et al. [14], and secondly for a field scale natural gas jet fire considered by Cook at al. [15]. The application of participating media models to jet fires at these two diverse scales is a severe test of the generality of the respective models. In deriving the results for radiative fluxes given below, radiation calculations were performed using the discrete transfer method as a post-process on converged and grid-independent numerical calculations of the combusting flow fields. In the case of the laboratory scale flame, use of the discrete transfer method in conjunction with 192 rays per receiver was found to result in converged values for the radiative fluxes. This number of rays was consequently used for all receiver locations and participating media models. A staggered ray distribution [16] was also employed over the hemispherical surface considered about each receiver in order to improve numerical convergence characteristics and run times, although this approach was applied equally in all cases. For the field scale fire, a ray adaption technique was also used to improve model run times since the remote location of receivers, and hence the large numbers of rays required, meant that without such an approach the ray tracing algorithm would have dominated model run times. This meant that different numbers of rays were employed in conjunction with 


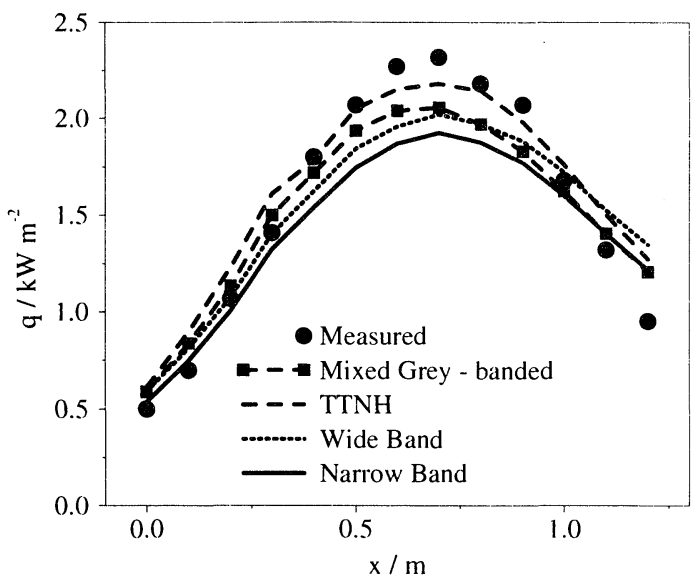

FIGURE 3 Variation of radiative fluxes with vertical distance for the laboratory scale fire.

each participating media model, although, as will be seen later, the total number of rays used was similar in each case.

The laboratory scale flame considered by Baillie et al. [14] was stabilised on a release of methane issuing vertically from a $8.6 \mathrm{~mm}$ internal diameter circular pipe at a mean exit velocity of $20 \mathrm{~m} \mathrm{~s}^{-1}$. A second concentric pipe of $23 \mathrm{~mm}$ diameter was also employed so that a small co-flow of methane through the annular gap between the two pipes could be used to rim-stabilise the main flame. The resulting flame was 1.1 to $1.2 \mathrm{~m}$ in height. Thermal radiation levels about this flame were measured using a radiometer with a $150^{\circ}$ field of view and by tracking the radiometer vertically $(x)$ at a horizontal distance of $0.4 \mathrm{~m}$ from the release, with its normal pointing towards the flame, and horizontally $(r)$ at the height of the pipe exit with its normal pointing vertically upwards. The results obtained are shown, respectively, in Figures 3 and 4.

The field scale fire [15] considered was formed by a vertical, underexpanded release of natural gas at $65.1 \mathrm{~kg} \mathrm{~s}^{-1}$ from a $16.2 \mathrm{~m}$ high, $385 \mathrm{~mm}$ internal diameter, circular pipe. This fire, which was approximately $60 \mathrm{~m}$ in length, was subjected to a cross-wind with a velocity at $9 \mathrm{~m}$ above ground level of $6.4 \mathrm{~m} \mathrm{~s}^{-1}$. Radiation measurements about the fire were made using radiometers that were positioned downwind $(x)$ with their normals orientated towards the fire in order to maximise received radiative fluxes. The received fluxes obtained are given in Figure 5.

Figures 3 and 4 show results for the laboratory scale flame obtained using the four participating media models that yield converged predictions in conjunction with the discrete transfer method, with Table 1 showing model run times, again in terms of a speed-up factor relative to results obtained using the narrow band approach. Values of $S$ given in Table 1 are averages over all receiver locations. The level of agreement between each of the four models and the experimental data displayed in the latter figures is satisfactory, with all the 


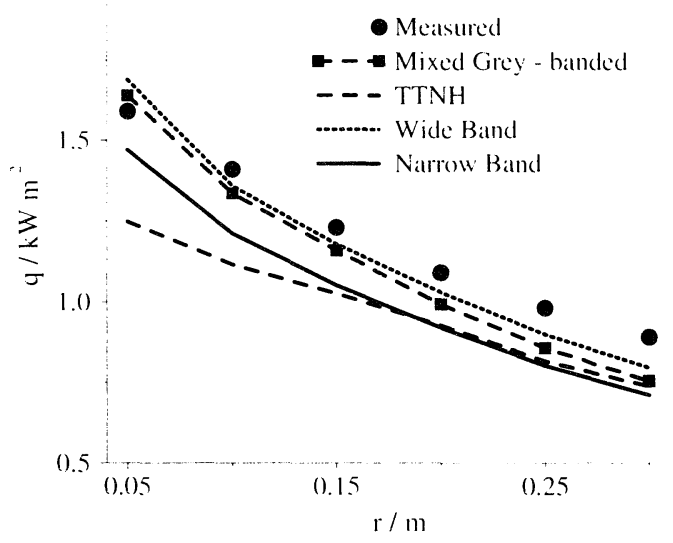

FIGURE 4 Variation of radiative fluxes with horizontal distance for the laboratory scale fire.

models being generally within $20 \%$ of observations. Agreement between predictions of the various models and the data is, however, largely dependent upon the accuracy of the fluid dynamic computations for the structure of the flame, and because of this these results cannot be used to assess the relative accuracy of the various participating media models. A more meaningful comparison is between predictions of the various models and results derived using the more accurate narrow band approach, which reveals that fluxes obtained using the banded grey gas and wide band models are within $13 \%$ of the narrow band results, whilst those of the TTNH approach are within $22 \%$. This level of performance may be considered adequate for most fire calculations. The results of Table 1 also demonstrate that in applying these models to real flame calculations, for this flame at least, the speed-up factors realised for the idealised, one-dimensional test case are not achieved in practice. In particular, the run times associated with the banded version of the mixed grey gas model and the TTNH approach are now an order of magnitude less than those achieved for the latter case. In addition, run times of the wide band model are now almost three times faster than those of the narrow band approach, primarily due to the fact that, compared to the one-dimensional test case, the number of homogeneous elements used to represent any non-homogeneous path has been dramatically reduced.

The results displayed in Figure 5 for the field scale fire tend to confirm the conclusions reached above for model accuracy. In particular, predictions of the banded grey gas and wide band models are within $10 \%$ of narrow band model results, although the TTNH approach consistently overpredicts the latter model by between 80 and $90 \%$. In real terms, however, this is only a problem close to the fire, as displayed in Figure 5. Compared to the laboratory scale flame, the run times of the various models, given in Table 1, are now more in line with those obtained for the one-dimensional test case, with the TTNH and mixed grey approaches being, respectively, one and two orders of magnitude faster than the narrow band model. 


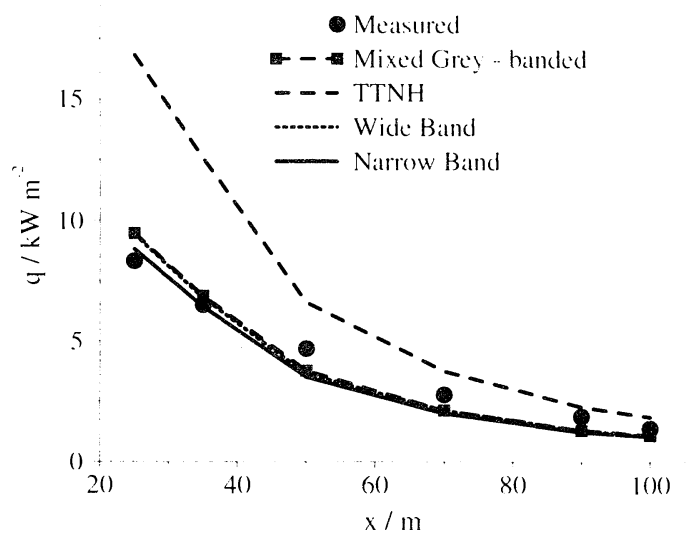

FIGURE 5 Variation of radiative fluxes downwind of the field scale fire.

The differences in model run times exhibited in the results of Table 1 may be attributed to the increasing dominance of the ray tracing algorithm within the discrete transfer method in increasingly complex geometric configurations. For the field scale flame, therefore, ray tracing was performed through a three-dimensional, Cartesian mesh. These computations are relatively straight forward, and as a consequence the speed-up factors are similar to those obtained for the one-dimensional test case. The only differences that do occur are for the wide band model where, as noted above, a significantly reduced number of control volumes was used along each ray, and for the TTNH approach, where ray tracing and the determination of effective pressure-path lengths and temperatures have a disproportionately large effect on run times. For the laboratory scale flame, the ray tracing algorithm was used in conjunction with an axisymmetric finite-volume mesh that expanded in the downstream and cross-stream directions. This mesh was also non-rectangular due to the use of a strean function formulation in solving the flow equations. As a consequence, ray tracing dominated all the radiative flux computations, and dramatically reduced all model run times relative to the narrow band approach.

Lastly, a limited number of computations were also performed using the temperature correction factor to the transmittance determined within the TTNH model suggested by Grosshandler and Modak [7, 10]. In all cases, the incorporation of this factor significantly reduced agreement between TTNH model results and those obtained using the narrow band approach. Further work to improve the applicability of this factor to the conditions encountered in computational fluid dynamic calculations of fires would be useful.

\section{CONCLUSIONS}

1. The implementation of simple participating media models within the discrete transfer method, based on the assumption of grey gas behaviour, must be treated with caution since 
radiative fluxes predicted using this approach do not converge as the representation of a nonhomogeneous path is refined.

2. In general, use of the discrete transfer method together with the banded grey gas, TTNH, wide and narrow band models yields satisfactory results for fire applications, with an adequate level of agreement between the various models, and with experimental data.

3. For computations of fires obtained in conjunction with Cartesian meshes, the TTNH and banded grey gas models give speed-up factors, respectively, of one and two orders of magnitude relative to the wide and narrow band approaches. The performance of all models relative to the narrow band approach is, however, dramatically reduced when more complex, non-Cartesian meshes are employed.

4. Results for the idealised test case indicate that only a relatively small number of homogeneous cells is required by some models to represent a non-homogeneous profile. This suggests that radiation calculations for fires could be performed on meshes that are more coarse than those generally used for the fluid dynamic computations. However, use of the wide band model should be treated with some caution due to the large number of control volumes required to give converged results. This situation can be improved through adaptations to the standard approach used in the present work, or through use of wide band scaling techniques. These methods will be considered in a separate publication.

\section{ACKNOWLEDGEMENT}

This paper is published by permission of BG Technology.

\section{REFERENCES}

1. Caulfield, M., Cook, D.K., Docherty, P. and Fairweather, M., "An Integral Model of Turbulent Jets in a Cross-Flow. Part 2 - Fires", Transactions I.Chem.E., 71: B4, 243$251,1993$.

2. Fairweather, M., Jones, W.P. and Lindstedt, R.P., "Predictions of Radiative Heat Transfer from a Turbulent Reacting Jet in a Cross-Wind", Combustion and Flame, 89: 1, 45-63, 1992.

3. Tien, C.L. and Lee, S.C., "Flame Radiation", Progress in Energy and Combustion Science, 8: 1, 41-59, 1982.

4. Lockwood, F.C. and Shah, N.G., "A New Radiation Solution Method for Incorporation in General Combustion Prediction Procedures", Eighteenth Symposium (International) on Combustion, pp. 1405-1414, The Combustion Institute, Pittsburgh, 1981.

5. Modak, A.T., "Radiation from Products of Combustion", Fire Research, 1: 4, 339-361, 1978/79. 
6. Truelove, J.S., "A Mixed Grey Gas Model for Flame Radiation", AERE-R8494, UKAEA, Harwell, 1976.

7. Grosshandler, W.L., "Radiative Heat Transfer in Nonhomogeneous Gases: A Simplified Approach", International Journal of Heat and Mass Transfer, 23: 11, 1447 $1459,1980$.

8. Edwards, D.K. and Balakrishnan, A., "Thermal Radiation by Combustion Gases", International Journal of Heat and Mass Transfer, 16: 1, 25-40, 1973

9. Modest, M.F., "The Weighted-Sum-of-Gray-Gases Model for Arbitrary Solution Methods in Radiative Transfer", Journal of Heat Transfer, 113: 3, 650-656, 1991.

10. Grosshandler, W.L. and Modak, A.T., "Radiation from Nonhomogeneous Combustion Products", Eighteenth Symposium (International) on Combustion, pp. 601-609, The Combustion Institute, Pittsburgh, 1981.

11. Edwards, D.K., "Molecular Gas Band Radiation", in Advances in Heat Transfer, eds. T.F. Irvine and J.P. Hartnett, Vol. 12, pp. 115-193, Academic Press, New York, 1976.

12. Goody, R.M., Atmospheric Radiation I: Theoretical Basis, Clarendon Press, Oxford. 1964.

13. Cumber, P.S., Fairweather, M. and Ledin, H.S., "Application of Wide Band Radiation Models to Non-Homogeneous Combustion Systems ", International Journal of Heat and Mass Transfer, 41: 11, 1573-1584, 1998.

14. Baillie, S., Caulfield, M., Cook, D.K. and Docherty, P., "A Phenomenological Model for Predicting the Thermal Loading to a Cylindrical Vessel Impacted by High Pressure Natural Gas Jet Fires", Transactions I.Chem.E., 76: B1, 3-13, 1998.

15. Cook, D.K., Cumber, P.S., Fairweather, M. and Shemirani, F., "Modelling Free and Impacting Underexpanded Jet Fires", I.Chem.E. Symposium Series, No. 141, pp. $127-$ 138, The Institution of Chemical Engineers, Rugby, 1997.

16. Cumber, P.S., "Ray Effect Mitigation in Jet Fire Radiation Modelling", to be published. 